\title{
Multiple well-differentiated cardiac liposarcoma with a concomitant myocardial lipoma: A case report
}

\author{
FANLEI KONG, WEI ZHANG and QIYONG GUO \\ Department of Radiology, Shengjing Hospital of China Medical University, Shenyang, Liaoning 110004, P.R. China
}

Received April 16, 2018; Accepted August 20, 2018

DOI: $10.3892 /$ mco.2018.1741

\begin{abstract}
A cardiac lipoma and a cardiac liposarcoma are very uncommon conditions; however, the simultaneous development of the two is extremely rare. In addition, the symptoms of these conditions are not evident in the vast majority of cases, making them easily missed during diagnosis. The present case report describes a case of well-differentiated cardiac liposarcoma with a concomitant myocardial lipoma that was misdiagnosed as multiple cardiac lipomas. The ultrasound, computed tomography, and magnetic resonance imaging presentations of the tumour were described. In addition, relevant literature regarding these two types of tumours was reviewed and compared in order to help improve the identification of these types of tumours. This, in turn, may be beneficial in the selection of more appropriate examination and treatment strategies.
\end{abstract}

\section{Introduction}

Primary cardiac tumours are very uncommon, and approximately $75 \%$ of primary cardiac tumours are benign. In adults, myxoma is the most common type of primary benign cardiac tumours, followed by lipoma, fibroma, and teratoma $(1,2)$. The incidence of primary cardiac lipomas is only $0.17-0.19 \%$ based on autopsy reports (3). Cardiac lipomas account for $8.4 \%$ of all primary cardiac tumours; they have no gender or age predominance (4) and they vary significantly in size (3). Most cardiac lipomas arise from the endocardium or the epicardium, while very few develop in the myocardial layer. Although there have been reports of cardiac lipomas developing in both the atria

Correspondence to: Professor Qiyong Guo, Department of Radiology, Shengjing Hospital of China Medical University, 36 Sanhao Street, Shenyang, Liaoning 110004, P.R. China

E-mail: 437201292@qq.com

Abbreviations: CT, computed tomography; MRI, magnetic resonance imaging; $\mathrm{H} \& \mathrm{E}$, haematoxylin and eosin

Key words: cardiac lipoma, cardiac liposarcoma, echocardiography, CT, MRI and the ventricles, they generally tend to develop in the right atrium (4).

A cardiac lipoma should be distinguished from cardiac liposarcoma. Liposarcoma was first reported by Virchow in 1857. Despite liposarcoma being the most common cancer of mesenchymal origin in adults, $75 \%$ of liposarcoma cases occur in deep muscle tissue of the limbs, $20 \%$ occur in the retroperitoneum, and the remainder occur in other sites. Mediastinal liposarcoma is extremely rare $(5,6)$; primary mediastinal liposarcoma accounts for only $0.13-0.75 \%$ of mediastinal cancers, and most occur in the posterior mediastinum (6). Cardiac liposarcoma is even rarer and account for approximately $1 \%$ of all primary cardiac malignant tumours $(2,7,8)$. Similar to cardiac lipomas, cardiac liposarcoma has no gender predominance (9), and most also occur in the right atrium (7).

The symptoms of cardiac lipoma and liposarcoma are not obvious in the vast majority of cases, making them easy to be missed in diagnosis. Moreover, cardiac lipoma and cardiac liposarcoma (especially well-differentiated liposarcoma) are sometimes difficult to distinguish, and they are easily misdiagnosed.

This paper presents a case of multiple well-differentiated cardiac liposarcoma with a concomitant myocardial lipoma. We describe the patient's presentation including details regarding their ultrasound, computed tomography (CT), and magnetic resonance imaging (MRI) findings, to help improve the diagnosis and treatment of this condition. To our knowledge the coexistence of these two conditions has never been reported in the literature.

\section{Case report}

A 49-year-old Chinese male experienced idiopathic chest and back pain for $>20$ days and thus sought treatment at the local hospital. Echocardiography indicated space-occupying masses in the right atrium and pericardium. The patient was recommended to seek further diagnosis and treatment at our hospital. The patient had a history of an ischemic stroke that occurred 2 years prior, but otherwise he had no past medical or family history. After admission, a physical examination revealed murmurs that changed with position at the left edge of the sternum, between ribs 4 and 5. Laboratory examination did not reveal any obvious abnormalities.

At our hospital, transthoracic echocardiography indicated a very large solid mass $(162 \times 105 \mathrm{~mm})$ in the pericardium located primarily in the posterior of the heart and surrounding 
it, with an inhomogeneous hypoechoic interior accompanied with hyperechoic bands. Deformation of the cardiac apex was accompanied by a local protrusion $(32 \times 28 \mathrm{~mm})$, inside which the blood flow signal was visible. Moderately hyperechoic lumps $(48 \times 31 \mathrm{~mm})$ were visible in the right atrium (Fig. 1). The mass was connected to the lateral wall of the right atrium near the atrioventricular ring by a stem like connection, which moved with the cardiac cycle and with mild tricuspid valve regurgitation. Further $\mathrm{CT}$ scanning showed a dense mass with highly homogeneous fat inside the pericardium, inside which a few slightly high-density slivers were visible. In the right atrium, a dense fatty mass with lobulation was visible, and the boundary between the mass and the right atrial wall was unclear (Fig. 2). After obtaining written informed consent from the patient, a free contrast-enhanced cardiac MRI examination was performed. The results showed that the pericardial mass was composed of fat, and its interior was not enhanced. The mass in the right atrium was also composed of fat, and an envelope was visible in its periphery. The mass was continuous with the lateral right atrial wall and it oscillated with blood flow. The local protrusions in the cardiac apex had weaker movement, and a narrow neck-like connection with the left ventricle was visible (Fig. 2). Considering the medical history of the patient, the various examinations performed, and additional discussion, we made a diagnosis of right atrial and pericardial lipomas and apical diverticula. The cardiac surgeon decided to surgically remove the cardiac lipomas.

With the patient on extracorporeal circulation, a median sternotomy was performed, and the pericardium was opened. A very large fatty mass $\left(20.4 \times 13.5 \times 5 \mathrm{~cm}^{3}\right)$ was visible between the bottom of the heart and the diaphragm and was continuous with the cardiac apex. In addition, a fatty mass $\left(6 \times 3.5 \times 2 \mathrm{~cm}^{3}\right)$ was visible in the right atrial wall protruding into the atrium. The fatty masses in the pericardium and right atrium were completely resected. After opening the right atrial wall, multiple small fatty masses (the size of the larger of these masses was $3 \times 2 \times 1.5 \mathrm{~cm}^{3}$ ) were visible in the right atrial wall; these were also resected. The pericardial membrane naturally repaired the right atrium, and indwelling drainage tubes were placed in the pericardium and behind the sternum.

Postoperative pathological examination revealed atypical lipomas (well-differentiated liposarcoma) in the pericardium and the right atrial wall (Fig. 3), and a myocardial lipoma was identified in the right atrium (Fig. 3). The patient had a large amount of postoperative pericardial drainage, and an open chest surgery was performed the following night. The wound exhibited slight blood seepage, and once haemostasis was confirmed, the undiscovered active bleeding was investigated. The patient was postoperatively given an infusion of red blood cells and plasma to alleviate anaemia and coagulation. The patient was discharged on postoperative day 9 , the symptoms disappeared, and follow-up after 1 year did not reveal recurrence.

This study was conducted with written informed consent from the patient.

\section{Discussion}

Pathologically, a cardiac lipoma can be categorized into two types: Lipomatous hypertrophy of the interatrial septum and

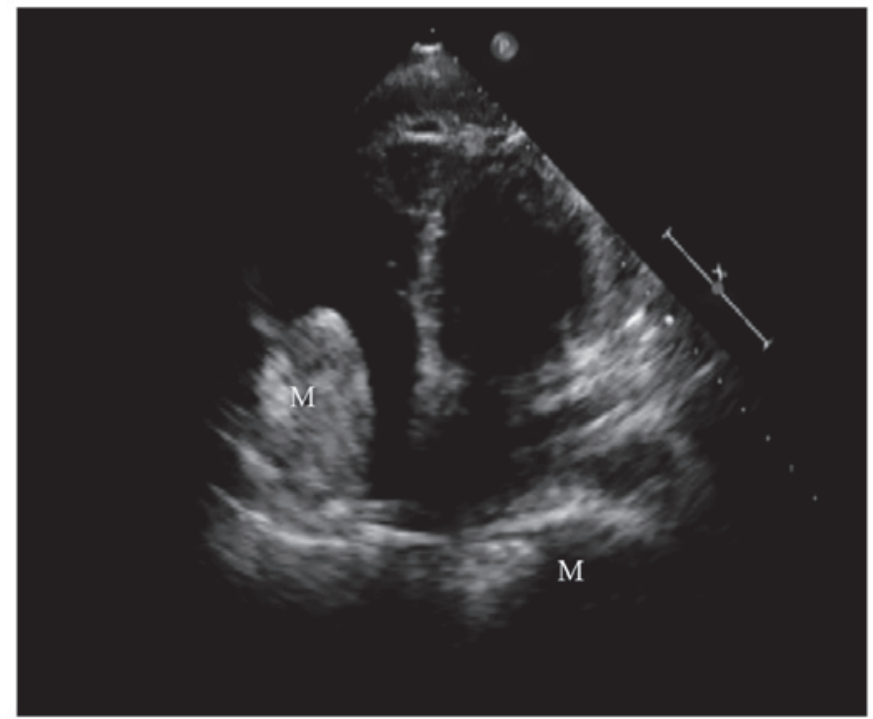

Figure 1. Transthoracic echocardiography revealed a massive solid hypoechoic mass inside the pericardium. Lump-like, slightly hyperechoic masses are visible in the right atrium. M, mass.

a true lipoma (1,3). Lipomatous hypertrophy tends to develop in the interatrial septum and usually develops continually with the epicardial fat. A true lipoma, on the other hand, is a mass composed of encapsulated adipose tissue, usually mature adipocytes $(3,4)$. When a lipoma invades the myocardium, the tumour is known as a myocardial lipoma. If fibrous components appear, then the tumour is known as a fibrolipoma. If the tumour contains only adipocytes, it is known as a lipoma (1). In our patient, the mass in the right atrium had invaded the myocardium, and proliferating adipose tissue was observed in the myocardium by microscopy. Accordingly, the pathological diagnosis was myocardial lipoma.

The criteria for liposarcoma diagnosis and classification are based on the pathological criteria established by the WHO (10). Liposarcomas can be divided into four subtypes: Well-differentiated liposarcoma (or atypical lipoma), myxoid/round cell liposarcoma, pleomorphic liposarcoma, and dedifferentiated liposarcoma. Well-differentiated liposarcoma accounts for 40-45\% of liposarcoma cases and they usually have a very low degree of malignancy (2). A lipomatous presentation is the most common presentation among the various types of well-differentiated liposarcoma (6). Local lesion cells with slightly enlarged nuclei were visible in the masses in the pericardium and right atrial wall of our patient, and the pathological diagnosis was lipomatous well-differentiated liposarcoma (or atypical lipoma).

Cardiac lipomas are usually asymptomatic due to their slow rates of growth and softness $(11,12)$, and are usually serendipitously discovered in autopsies or examinations for other reasons. Cardiac liposarcoma tends to invade neighbouring organs, so they are more likely to cause symptoms than lipomas, which only compress surrounding organs (6). The symptoms of cardiac lipoma and liposarcoma are generally similar, and patients often present with fatigue, dyspnoea, syncope, chest discomfort, chest pain, palpitation, and even sudden death $(3,11)$. The appearance of symptoms is usually determined by the location and size of the tumour. If the 

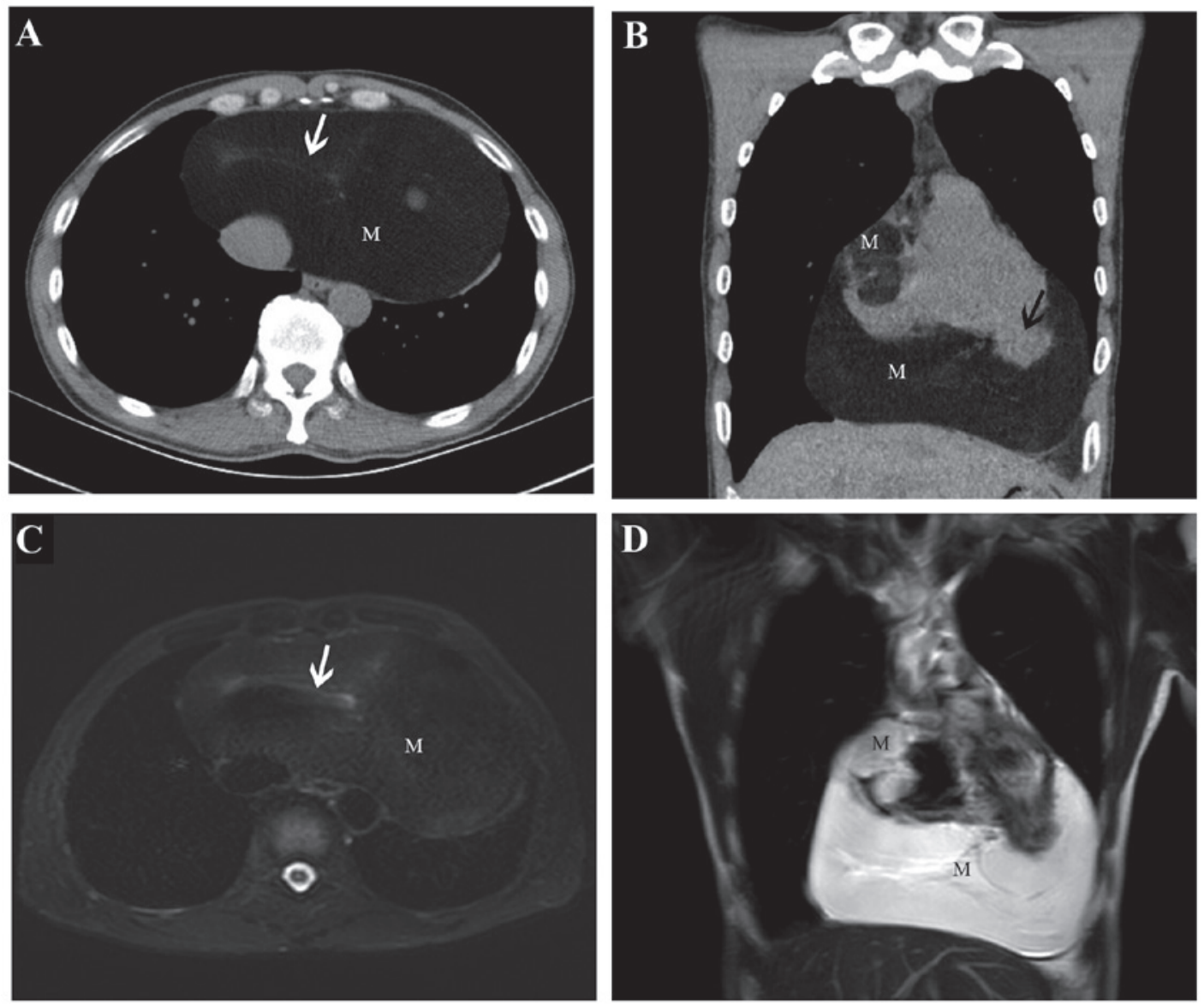

Figure 2. Radiology examination results. (A and B) A multi-layer spiral computed tomography scan revealed that the pericardium contained (A) a dense fatty mass with a small number of thin slivers (white arrow). A dense fatty mass with lobulation was observed in the right atrium. (B) Apical diverticula were also present (black arrow). (C and D) Magnetic resonance imaging scanning revealed that the pericardium contained (C) a mass with fatty signal accompanied by low-signal slivers (white arrow). The mass exhibited low signal on the fat suppression sequence. (D) Irregular lump-like signals were observed in the right atrium near the atrioventricular groove. M, mass.

A

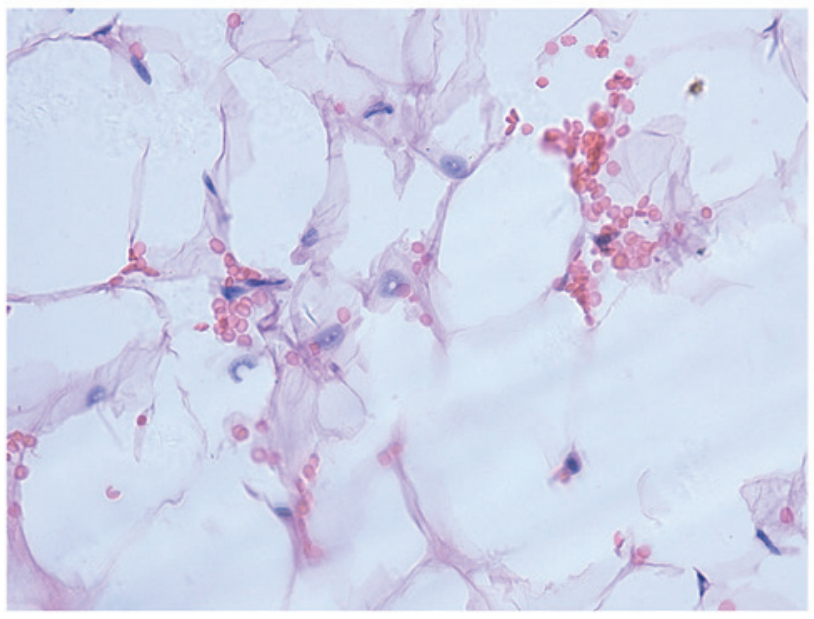

B

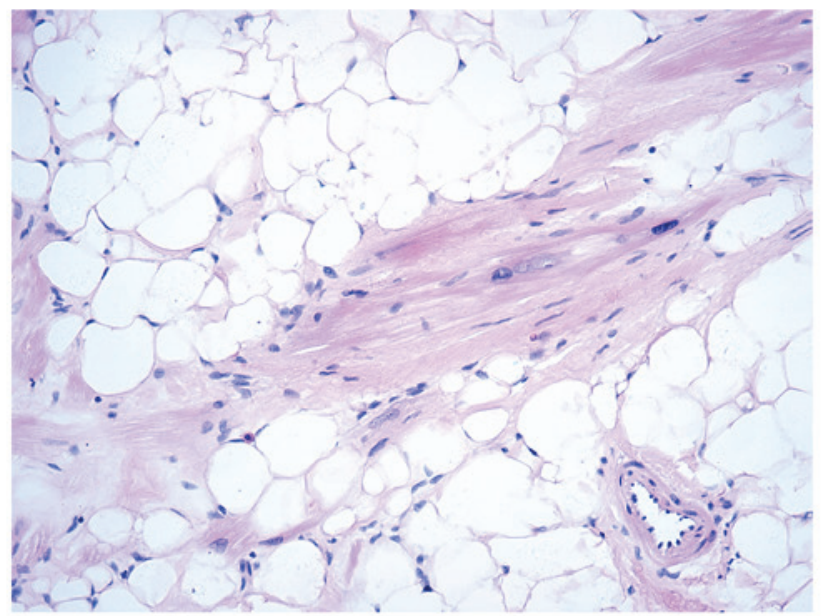

Figure 3. Pathological examination results. (A) Microscopy of the pericardium interior and the right atrial wall revealed a sheet-like arrangement of fat cells. The nuclei of local lesion cells were slightly enlarged (H\&E staining; magnification, x40). (B) Microscopy of the right atrium interior revealed proliferating adipose tissue (H\&E staining; magnification, x20). H\&E, haematoxylin and eosin.

tumour is located under the pericardium, it may grow to a large size but remain asymptomatic; such tumours are often clinically discovered at a late stage. Many pericardial lipomas can cause dyspnoea by occupying the air spaces of the lungs or compressing the heart. Chest pain can be caused by compression of the coronary arteries by the tumour. Tumours located near the valves may lead to valve regurgitation. Finally, myocardial lipomas that interfere with the conduction system 
can lead to arrhythmias $(12,13)$. Our patient had a myocardial lipoma in the right atrium, and as a result, exhibited mild tricuspid valve regurgitation and heart murmur on physical examination. The patient's chest and back pain may have been caused by the atypical lipoma compressing the coronary arteries.

Conventional X-ray examinations cannot usually achieve an accurate diagnosis of cardiac tumours. Thus, transthoracic echocardiography has become an extremely valuable method for the initial discovery and diagnosis of cardiac tumours due to its high accuracy and non-invasiveness. On transthoracic echocardiography, pericardial lipomas usually present a relatively anechoic structure. In contrast, lipomas in the heart cavity have an echoic presentation (12). However, CT and MRI, and especially contrast-enhanced examination, are the most important and valuable methods for providing information on tumour size, location, and composition. Lipomas are often easily distinguishable by CT because they are composed of low-density tissue. On MRI, lipomas present with high signal intensity on T1- and T2-weightedimages, and the signal is significantly decreased when fat suppression sequences are used (14). Liposarcoma often presents with disordered density on conventional CT scanning. In addition, calcification is occasionally observed. On MRI, liposarcoma is often presented as primarily lipid signals with irregular mixed signal, and sometimes only present as a small number of soft tissue bands within the lipid signals. On enhanced CT and MRI, they often present as irregular enhancements (13). However, our patient presented with homogeneous adipose density or signal, and the well-differentiated liposarcoma in the pericardium only presented as a small number of bands with no definite enhancement observed. This suggests that CT and MRI are still insufficient for distinguishing between lipoma and well-differentiated liposarcoma. Consistently, the results of a study by Goto et al (7) also suggest that CT and MRI cannot distinguish between benign and malignant cardiac pathologies.

In addition to imaging examination, biopsy is often insufficient for the accurate diagnosis of lipomatous masses, especially for distinguishing between lipomas and well-differentiated liposarcoma. This difficulty arises because biopsy sample collection can only partially represent the degree of differentiation, and immunohistochemical and ultrastructural analysis cannot be completed with insufficient sample collection. Thus, accurate diagnosis often requires open surgery or pathological examination of resected tissue (6).

It is exactly because neither imaging nor biopsy can accurately diagnose lipomatous masses, and because cases of sudden death have been reported, that we advocate for proactive surgical resection. This is also true for asymptomatic patients whose masses are accidently found, followed by patient management and determination of the prognosis based on postoperative pathological classification. According to the literature, cardiac lipoma patients that undergo surgical resection have good prognoses (15), and to our knowledge there are currently no case reports of recurrence. The prognosis for cardiac liposarcoma is primarily determined by pathological subtype and the range of safety of surgical resection. The recurrence rate is approximately $40 \%$, and the longest time to recurrence has been reported as 14 years post-operation (6).
The patient described in the present report did not undergo chemotherapy after successful surgical resection and exhibited no signs of recurrence when re-examined 1 year after surgery.

\section{Acknowledgements}

Not applicable.

\section{Funding}

No funding was received.

\section{Availability of data and material}

All data generated or analyzed during this study are included in this published article.

\section{Authors' contributions}

FK performed the majority of the experiments. WZ captured and assessed the images, and critically analysed the manuscript for important intellectual content. QG conceived the study and gave final approval of the version to be published. All authors read and approved the contents of the final draft of the manuscript.

\section{Ethics approval and consent to participate}

Written informed consent was obtained from the patient.

\section{Patient consent for publication}

Written informed consent was obtained from the patient.

\section{Competing interests}

The authors declare that they have no competing interests.

\section{References}

1. Habertheuer A, Andreas M, Wiedemann D, Rath C and Kocher A: A rare case of obstructive right atrial lipoma. Ann R Coll Surg Engl 96: e39-e41, 2014.

2. Papavdi A and Agapitos E: Undiagnosed primary cardiac liposarcoma in an adult: A case report and review of the literature. Am J Forensic Med Pathol 34: 299-301, 2013.

3. Wu S, Teng P, Zhou Y and Ni Y: A rare case report of giant epicardial lipoma compressing the right atrium with septal enhancement. J Cardiothorac Surg 10: 150, 2015.

4. Khoueiry G, Abi Rafeh N, Waked A, Abdo A, Azab B, Asgarian KT and Snyder ST: Left atrial appendage lipoma: An unusual location of cardiac lipomas. Echocardiography 28: E91-E93, 2011.

5. Ortega P, Suster D, Falconieri G, Zambrano E, Moran CA Morrison C and Suster S: Liposarcomas of the posterior mediastinum: Clinicopathologic study of 18 cases. Mod Pathol 28: 721-731, 2015.

6. Steger CM: Primary liposarcoma of the heart. BMJ Case Rep 2011: pii: bcr0320114013, 2011.

7. Goto T, Ohte N, Tani T, Suda H and Kimura G: Malignant nature of cardiac liposarcoma revealed by fluorine-18 fluorodeoxyglucose positron emission tomographic imaging. Intern Med 51: 1367-1370, 2012.

8. Grebenc ML, Rosado de Christenson ML, Burke AP, Green CE and Galvin JR: Primary cardiac and pericardial neoplasms: Radiologic-pathologic correlation. Radiographics 20: 1073-1103, 2000 . 
9. Araoz PA, Eklund HE, Welch TJ and Breen JF: CT and MR imaging of primary cardiac malignancies. Radiographics 19: $1421-1434,1999$

10. Fletcher CDM, Unni KK and Mertens F: World Health Organization classification of tumors. In: Pathology and genetics of tumors of soft tissue and bone. Kleihues P and Sobin LH (eds.) IARC, Lyons, pp35-46, 2002.

11. Zwolinski R, Ammer A, Walczak A and Jaszewski R Intrapericardial lipoma: Diagnosed unexpectedly and resected during coronary artery bypass surgery. Interact Cardiovasc Thorac Surg 11: 211-212, 2010

12. Vijay SK, Dwivedi SK, Chandra S and Saran RK: Giant intrapericardial lipoma: An unusual cause of dyspnoea. Indian Heart J 65: 104-106, 2013.

13. Zhu H, Wang M, Feng D, Feng Y, Ren Y, Chen J, He Y and Yuan J: Ultrasonography, X-ray and CT imaging findings of a giant pericardial lipoma: Imaging diagnosis and review of the literature. Oncol Lett 7: 195-198, 2014.
14. van Beek EJ, Stolpen AH, Khanna G and Thompson BH: CT and MRI of pericardial and cardiac neoplastic disease. Cancer Imaging 7: 19-26, 2007.

15. Girrbach F, Mohr FW and Misfeld M: Epicardial lipoma-a rare differential diagnosis in cardiovascular medicine. Eur J Cardiothorac Surg 41: 699-701, 2012.

This work is licensed under a Creative Commons Attribution-NonCommercial-NoDerivatives 4.0 International (CC BY-NC-ND 4.0) License. 